\title{
PRevention of INCisional hernia after liver transplantation (PRINC trial): study protocol for a randomized controlled trial
}

\author{
Daniela Kniepeiss ${ }^{1,2+}$, James Elvis Waha ${ }^{3 \dagger}$, Thomas Auer ${ }^{3}$, Andrea Berghold ${ }^{4}$ and Peter Schemmer ${ }^{1,2,5^{*}}$
}

\begin{abstract}
Background: Incisional hernia is a common complication after liver transplantation with an incidence of 5 to $46 \%$. Concerning non-transplant patients, a recently published meta-analysis describes a reduction of the incidence of incisional hernia of up to $85 \%$ due to prophylactic mesh replacement in elective, midline laparotomy. The aim of our study is to show a reduction of the incidence of incisional hernia after liver transplantation with minimal risk for complication.
\end{abstract}

Methods/design: This is an unblinded, randomized controlled trial comparing time to incisional hernia over a period of 12 months between patients undergoing liver transplantation and standardized abdominal closure with or without prophylactic placement of Phasix ${ }^{\mathrm{TM}}$ (Bard - Davol Inc., Warwick, RI, USA) mesh in an onlay position. As we believe that the mesh intervention is superior to the standard procedure in reducing the incidence of hernia, this is a superiority trial.

Discussion: The high risk for developing incisional hernia following liver transplantation might be reduced by prophylactic mesh placement. Immunosuppressed patients are at high risk for developing surgical-site infections. We chose a mesh which has anti-inflammatory properties and is fully resorbed after 18 months.

Trial registration: ClinicalTrials.gov, ID: 03222102. Registered retrospectively on 17 July 2018. Protocol version 1.4, 7 October 2018.

Keywords: Incisional hernia, Liver transplantation, Prophylactic mesh, Long-term absorbable mesh

\section{Background}

Incisional hernia is a common complication after liver transplantation with an incidence of 5 to $46 \%$ [1-12]. The high incidence rate is due to the very reduced general condition of the patients at the time of operation and is also dependent on the surgical technique, the materials used and the experience of the surgeon [13]. The essential immunosuppressive therapy after transplantation has a negative impact on wound healing and leads to the formation of dysfunctional scar tissue.

Artificial mesh is commonly used in ventral incisional hernia repair as described in the literature, but there is yet

\footnotetext{
* Correspondence: Peter.schemmer@medunigraz.at

${ }^{\dagger}$ Daniela Kniepeiss and James Elvis Waha contributed equally to this work.

${ }^{1}$ General, Visceral and Transplant Surgery, Transplant Center Graz, Medical University of Graz, Graz, Austria

${ }^{2}$ Transplant Center Graz, Medical University of Graz, Graz, Austria

Full list of author information is available at the end of the article
}

no scientific paper on the use of prophylactic mesh placement at the time of transplantation. The main reason, therefore, is that immunosuppressive therapy and artificial mesh implantation seem to be contradictory due an increased risk of complications.

Concerning non-transplant patients, a recently published meta-analysis by Borab et al. describes a reduction of the incidence of incisional hernia of up to $85 \%$ due to prophylactic mesh replacement in elective, midline laparotomy [14].

The development of Phasix ${ }^{\mathrm{Tm}}$ (Bard - Davol Inc., Warwick, RI, USA), an absorbable mesh, has given reason to conduct a study aiming at improving the healing process and stability of the abdominal wall. The mesh consists of suture material, which has proven reliability for many years and is characterized by an extremely long absorption rate of 12 to 18 months.

(C) The Author(s). 2019 Open Access This article is distributed under the terms of the Creative Commons Attribution 4.0 International License (http://creativecommons.org/licenses/by/4.0/), which permits unrestricted use, distribution, and 
Phasix $^{\text {max }}$ (Bard - Davol Inc., Warwick, RI, USA) is a fully resorbable monofilament mesh consisting of poly-4hydroxybutyrate (P4HB) (CE-certified) and is licensed for the treatment of hernias. $\mathrm{P} 4 \mathrm{HB}$ is degraded by hydrolysis to the monomer 4-hydroxybutyrate $(4-\mathrm{HB})$ which is a naturally occurring metabolite in humans. $\mathrm{P} 4 \mathrm{HB}$ has been in clinical use since 2007 as the suture material Monomax ${ }^{\circ}$ (Braun). Phasix ${ }^{\text {Ti }}$ (Bard - Davol Inc., Warwick, RI, USA) is comparable in repair strength to non-resorbable conventional mesh. It resorbs after 12 to 18 months and is gradually replaced by the host's own tissue [15].

In comparison to other fully resorbable meshes, Phasix ${ }^{\mathrm{Tm}}$ (Bard - Davol Inc., Warwick, RI, USA) shows higher stability over an even longer period. Particularly with regard to the stability during the first few weeks the material is strong enough to bridge the initial phase of healing. The phase of degradation is constant and restricted [16-20].

Preliminary results of a study conducted on rats at the University of Tennessee Health Science Center, Memphis, TN, USA showed a distinct decrease of methicillinresistant Staphylococcus aureus (MRSA)-infected Phasix ${ }^{\text {Tx }}$ (Bard - Davol Inc., Warwick, RI, USA) mesh compared to other biological implants, which emphasizes its value for use on immunosuppressed patients even more [21].

\section{Methods/design}

\section{Aim of the study}

The aim of our study is to show a reduction in the incidence of incisional hernia after liver transplantation with minimal risks for complications. We believe that the mesh intervention is superior to the standard procedure in reducing the incidence of hernia; therefore, it is a superiority trial.

\section{Inclusion and exclusion criteria}

Patients undergoing liver transplantation due to endstage liver diseases at the Department of Surgery, Division of Transplantation Surgery, Medical University of Graz will be included after written informed consent has been obtained.

\section{Inclusion criteria}

- Age $>18$ years

- First liver transplantation

- Written informed consent provided

\section{Exclusion criteria}

- Combined organ transplant

- Revision surgery after liver transplantation

- Previous liver transplantation
- Patients with a history of previous median laparotomy or transverse upper laparotomy, with a scar length of more than $15 \mathrm{~cm}$

- Pre-existing abdominal hernia, except umbilical

- Any signs of inflammation on the abdominal wall before transplantation

\section{Endpoint \\ Primary endpoint}

The primary endpoint of the study is the comparison of the time to incisional hernia over a period of 12 months between the two groups of patients with and without prophylactic mesh placement. Definition of incisional hernia: any detectable defect in the abdominal wall with intra-abdominal contents protruding beyond the aponeurosis [21].

\section{Secondary endpoint}

- Time to incisional hernia over a period of 24 months between the two intervention groups

- Rate of complications related to mesh placement, e.g., hematoma, seroma, chronic pain, wound dehiscence, mesh infections, mesh removal

\section{Further parameters}

- Date of birth

- Child-Pugh score and MELD score prior to liver transplantation

- Immunosuppression used

- Body Mass Index (BMI), height and weight

- Anamnesis of nicotine

- Diabetes mellitus

- Hepatocellular carcinoma

- Systemic corticosteroid therapy prior to transplantation

- Prior surgery of abdomen

- American Society of Anesthesiologists (ASA) classification

\section{Trial interventions}

This is a randomized controlled trial comparing the incidence of incisional hernia between patients undergoing liver transplantation with or without prophylactic mesh placement. The randomization is done using the "Randomizer for clinical trials" from the Institute for Medical Informatics, Statistics and Documentation, Medical University of Graz, Austria . A 1:1 randomization will be performed and patients will be assigned with a randomization number. 


\section{Patient education and study enrollment}

As soon as a patient is listed for liver transplantation and no exclusion criteria apply, the patient will be informed about the study (Figs. 1 and 2, Additional file 1 Standard Protocol Items: Recommendations for Interventional Trials (SPIRIT) Figure). When a patient signs the written informed consent, they are enrolled in the study. Afterwards the initial condition of the patient will be assessed and the case report form (CRF) will be filled in. The status of the abdominal wall and the existence of risk factors for development of hernia after liver transplantation will be evaluated (see "Section 4.3"). The patients will be randomly allocated to either the standard of care or the intervention group. To reach target sample size, all patients who will receive a liver transplantation at our center are in ambulant treatment before our study team makes contact with them and comprehensive participant enrollment can then be performed.

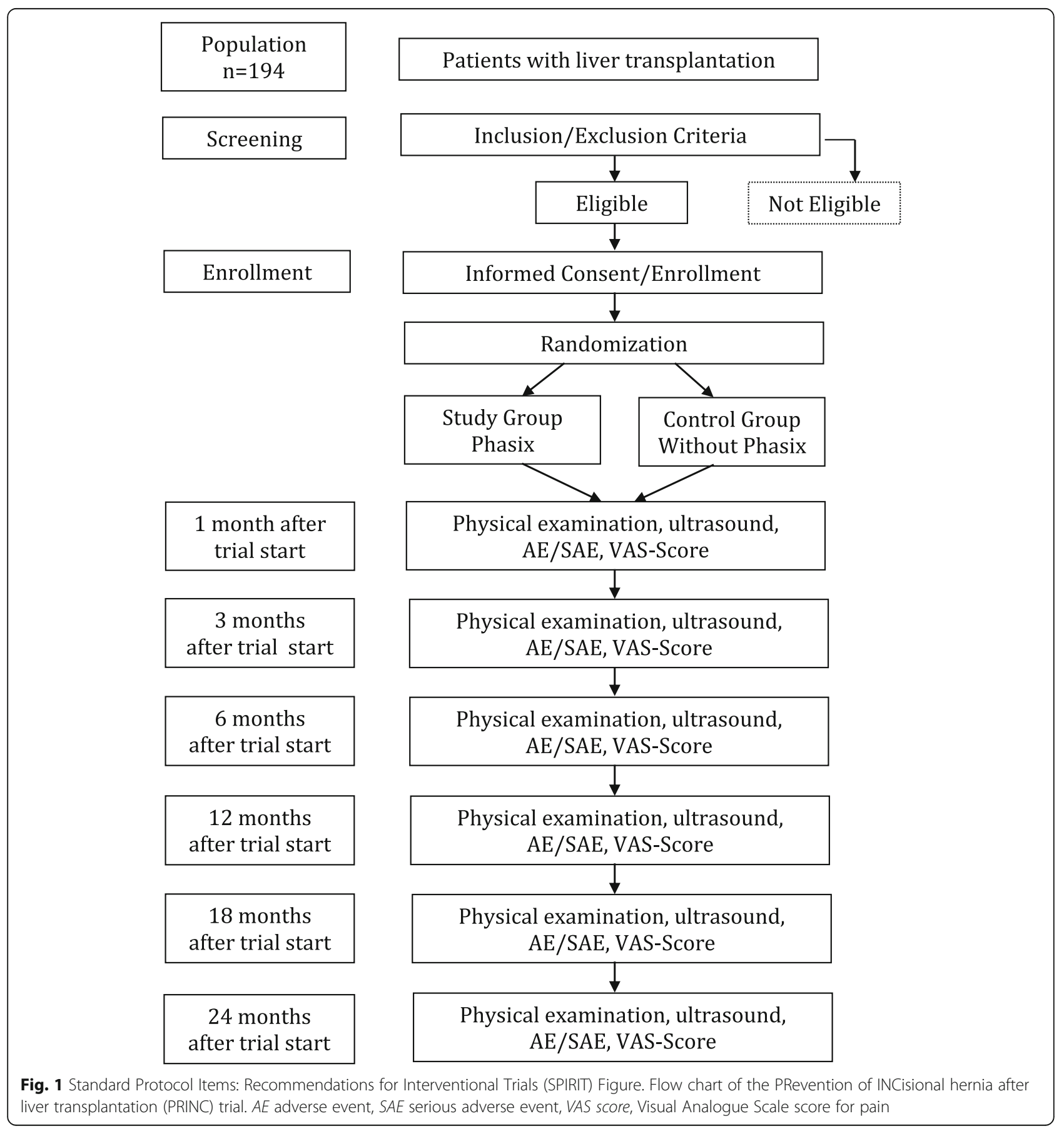




\begin{tabular}{|c|c|c|c|c|c|c|c|c|c|}
\hline \multirow[b]{3}{*}{ TIMEPOINT** } & \multicolumn{9}{|c|}{ STUDY PERIOD } \\
\hline & \multirow{2}{*}{$\frac{\text { Enrolment }}{-t_{1}}$} & \multirow{2}{*}{$\begin{array}{c}\text { Randomization } \\
\text { Transplantation }\end{array}$} & \multirow[b]{2}{*}{0} & \multicolumn{5}{|c|}{ Post-allocation (months) } & \multirow{2}{*}{$\frac{\text { Close-out }}{24}$} \\
\hline & & & & 1 & 3 & 6 & 12 & 18 & \\
\hline \multicolumn{10}{|l|}{ ENROLMENT: } \\
\hline \multirow{2}{*}{$\begin{array}{l}\text { Eligibility screen } \\
\text { Informed consent }\end{array}$} & $\mathrm{X}$ & & & & & & & & \\
\hline & $X$ & & & & & & & & \\
\hline Allocation & & $\mathrm{X}$ & & & & & & & \\
\hline \multicolumn{10}{|l|}{ INTERVENTIONS: } \\
\hline Mesh Placement & & $\mathrm{X}$ & & & & & & & \\
\hline \multicolumn{10}{|l|}{ ASSESSMENTS: } \\
\hline $\begin{array}{c}\text { Baseline Data } \\
\text { (number of transplantation, } \\
\text { preexisting median or } \\
\text { transverse laparotomy...) }\end{array}$ & $\mathrm{X}$ & & & & & & & & \\
\hline $\begin{array}{r}\text { (Indication for } \\
\text { transplantation, height, } \\
\text { weight, diabetes mellitus, } \\
\text { corticoid therapy...) }\end{array}$ & $X$ & & & & & & & & \\
\hline \multicolumn{10}{|l|}{$\begin{array}{r}\text { Intraoperative data } \\
\text { (Duration, number of } \\
\text { erythrocyte transfusion, } \\
\text { complications...) }\end{array}$} \\
\hline \multicolumn{10}{|l|}{$\begin{array}{r}\text { Post-surgical data } \\
\text { (Duration of invasive } \\
\text { ventilation, } \\
\text { complications...) }\end{array}$} \\
\hline $\begin{array}{l}\text { Post-surgical visits } \\
\text { (detection of hernia, signs } \\
\text { of infection or seroma, } \\
\text { image documentation) }\end{array}$ & & & & $\mathrm{X}$ & $\mathrm{X}$ & $X$ & $\mathrm{X}$ & $X$ & $\mathrm{X}$ \\
\hline
\end{tabular}

Fig. 2 Template of content for the schedule of enrollment, interventions and assessments (Standard Protocol Items: Recommendations for Interventional Trials. (SPIRIT) Figure

\section{Surgical technique}

Surgical access is through a midline laparotomy with right lateral extension. Liver transplantation is done using a standard or piggyback technique.

\section{Abdominal-wall closure technique - Standard group}

The closure of the J-shaped incision is started by placing two to three sutures of Ethibond 2-0 single stitches at the cranial end of the median part of the laparotomy and the far lateral end of the transverse part at a distance of 5-10 $\mathrm{mm}$ [22].

For the median laparotomy a full-thickness closure is performed using a PDS 2-0 double loop with intermittent single stitches using Ethibond 2 for augmentation $(3-4 \mathrm{~cm}$ distance). The posterior sheath of the transverse incision is then closed by a PDS 2-0 double loop, followed by a third PDS 2-0 double loop closing the anterior sheath of the transverse incision. The transverse incision suturing is augmented by intermittently placing Ethibond 2 single sutures (approximately 5-cm distance).
The wound is closed using vicryl 4-0 single stitches for the subcutis every $1-2 \mathrm{~cm}$. Skin closure is performed using staples.

\section{Abdominal-wall closure technique - Intervention group} After closure of the fascia in the above fashion a Phasix ${ }^{\text {Tm }}$ (Bard - Davol Inc., Warwick, RI, USA) mesh is positioned on the anterior rectus sheath with an overlap of $3 \mathrm{~cm}$ along the total length and width. Mesh fixation will be done using PDS 3-0 in simple interrupted sutures each at a $5-\mathrm{cm}$ distance along the margin of the mesh. A subcutaneous drainage will be used. For safety reasons the mesh is only implanted by experienced surgeons who have been instructed exactly on how to use it. Complications are handled according to the standards of our department.

\section{Postinterventional screening}

\section{Intraoperative data}

Duration of operation.

Number of platelet transfusion, erythrocyte transfusion and plasma transfusion. 
Operative complications (injuries to the intestine, bleeding).

\section{Post-surgical data}

Duration of invasive ventilation, complications, pulmonary infection, number of postoperatively required units of blood, days to first postoperative stool.

Postoperative complications: wound infection (according to the "New CDC Guideline for the Prevention of Surgical Site Infection") [23], hematoma needing surgical revision, total subcutaneous drainage flow, seroma after removal of drainage, revision surgery.

\section{Post-surgical visits}

Post-surgical visits will take place 1, 3, 6, 12, 18 and 24 months after liver transplantation and include the following specific examinations:

- Ultrasound examination: detection of clinically non-detectable hernia

- Signs of infection of the surgical site

- Signs of seroma

- Visual Analog Scale (VAS) score

- Image documentation

\section{Data management and statistical analysis}

Almost all patients after liver transplantation will receive their post-transplant follow-up in our center; therefore, patients will come for a routine check-up and at the same time this is the follow-up for the study. The data for patients will be collected until 24 months after liver transplantation or until they drop out (revision surgery, death, withdrawal, loss to follow-up). We will use a data monitoring committee (DMC).

\section{Planned statistics}

The primary analysis will be for the intention-to-treat (ITT) population including all patients randomized and having had the liver transplantation performed. A perprotocol analysis with all patients without major protocol violations will also be performed.

Demographic and clinical parameters will be displayed with mean and standard deviation and median, minimum and maximum for continuous variables. Categorical variables will be shown via absolute and relative frequency. Furthermore, differences between the groups are calculated using Student's $T$ test or the Mann Whitney $U$ test and the chi-squared test. The primary endpoint, time to incisional hernia over a period of 12 months, will be compared using a log-rank test. Additionally, we will also be plotting Kaplan-Meier survival curves by group looking at time to hernia development.

For sensitivity analysis a Cox regression analysis adjusted for age and MELD score will further be performed.
Subgroup analyses for immunosuppression therapy (everolimus)-specific differences are planned. A $p$ value $<0.05$ will be statistically significant. We perform our analyses with SPSS v25.0 (SPSS Inc., Chicago, IL, USA).

\section{Sample size estimation}

We estimate from the current data that about $20 \%$ of patients will develop an incisional hernia in the standard group 1 year after surgery. In the intervention group in which we use Phasix ${ }^{\mathrm{Tm}}$ (Bard - Davol Inc., Warwick, RI, USA) the prevalence of incisional hernia should drop to $5 \%$.

Considering these assumptions and a level of significance of $5 \%$ and a power of $80 \%$, we need to include 81 patients per group using a log-rank test. With regard to a drop-out rate of $10 \%, 178$ patients need be included in the study.

\section{Privacy}

All included patients are coded with continuous numbers (pseudonymization). The data to be evaluated are saved at the Department of Surgery, Division of General Surgery and Division of Transplantation Surgery and only labeled with this number, using an Excel sheet data base on a computer with access restriction, and then assessed. Only authorized persons have access to the original data. Data will be stored for 15 years. Plans for investigators and the sponsor to communicate trial results to participants and healthcare professionals include publications and participation at scientific meetings.

\section{Quality assurance}

The principles of the International Conference on Harmonization-Good Clinical Practice (ICH-GCP) guidelines [23], the regulations of the Declaration of Helsinki [24] and local legal and regulatory requirements will be adhered to at all times during the trial period. Important protocol modifications will be managed according to the appropriate guidelines. Ancillary care or compensation to those who suffer harm from trial participation is covered by study-related insurance.

\section{Discussion}

Due to a high incidence of incisional hernia after liver transplantation, patients would profit to a great extent from hernia prevention. Borab et al. [14] showed the benefits of prophylactic mesh placement in elective midline laparotomy, lowering the risk for the need for a second operation due to reducing the incidence of developing an incisional hernia. From our perspective there is a high variance for the incidence of developing an incisional hernia after liver transplantation. This variance is due to the different types of incisions used for liver transplant surgery. The transverse incision with subcostal extension has a high risk for developing an 
incisional hernia. J-shaped incisions seem to be associated with a lower risk but are still unsatisfactory. Placing a long-term resorbable mesh in an onlay position might improve the stability of the tissue and reduce the forces on the scar during the healing process until the mechanical strength of the resorbable mesh is resolved after 12 months and the scar tissue is stable. From our point view we hypothesize that this technique will reduce the incidence of developing incisional hernia from an estimated $20 \%$ to $5 \%$.

For placement of the mesh the fascia needs to be dissected from the fatty tissue; this surgical step is associated with a minimally elevated risk for developing seroma, while the risk for surgical-site infection is reduced by the precise wound-closure technique and the choice of mesh material. The risk for the included patients is very low. The potential risks are wound-healing disorder, seroma, hematoma and mesh infection; all of which are evenly associated with the standard procedure.

\section{Trial status}

Recruitment started on 1 January 2018. The protocol version of the study protocol is 3.0 from 18 April 2017; the protocol version of this manuscript is 1.4, 7 October 2018. Recruitment will be approximately completed in December 2021.

\section{Additional file}

Additional file 1: Standard Protocol Items: Recommendations for Interventional Trials (SPIRIT) 2013 Checklist: recommended items to address in a clinical trial protocol and related documents*. (DOC $121 \mathrm{~kb}$ )

\section{Acknowledgements}

All authors read and approved the final manuscript.

\section{Authors' contributions}

DK and JW designed the study and wrote the manuscript and contributed hereby equally. TA designed the study and critically reviewed the manuscript. AB performed the statistical design. PS critically revised both the study design and the manuscript. All authors read and approved the final manuscript.

\section{Funding}

The study receives no funding. This trial was conducted with no external funding and received no other funding.

\section{Availability of data and materials}

There is no data available yet; patient enrollment started in January 2018.

\section{Ethics approval and consent to participate}

The application for ethics approval has been sent to the local Ethics Committee on 8 August 2018. Ethics approval was received on 31 March 2018. Any patient included in this study has to sign an informed consent.

\section{Consent for publication}

All authors give consent for publication. All authors read and approved the final manuscript.

\section{Competing interests}

Thomas Auer is working as a consultant for BD-BARD, Davol.

The authors declare that they have no competing interests.

\section{Author details}

1General, Visceral and Transplant Surgery, Transplant Center Graz, Medical University of Graz, Graz, Austria. ${ }^{2}$ Transplant Center Graz, Medical University of Graz, Graz, Austria. ${ }^{3}$ General, Visceral and Transplant Surgery, Medical University of Graz, Graz, Austria. ${ }^{4}$ Institute for Medical Informatics, Statistics and Documentation, Medical University of Graz, Graz, Austria. ${ }^{5}$ Department of General, Visceral and Transplant Surgery, Transplant Center Graz, Medical University of Graz, Auenbruggerplatz 29, 8036 Graz, Austria.

Received: 3 December 2018 Accepted: 29 May 2019

Published online: 20 June 2019

\section{References}

1. de Goede B, Eker HH, Klitsie PJ, van Kempen BJ, Polak WG, Hop WC, et al. Incisional hernia after liver transplantation: risk factors and health-related quality of life. Clin Transpl. 2014;28(7):829-36.

2. Gianchandani R, Moneva E, Marrero P, Alonso M, Palacios MJ, Del Pino JM, et al. Feasibility and effectiveness of laparoscopic incisional hernia repair after liver transplantation. Transplant Proc. 2011;43(3):742-4.

3. Gomez R, Hidalgo M, Marques E, Marin L, Loinaz C, Gonzalez I, et al. Incidence and predisposing factors for incisional hernia in patients with liver transplantation. Hernia. 2001;5(4):172-6.

4. Kahn J, Muller H, Iberer F, Kniepeiss D, Duller D, Rehak P, et al. Incisional hernia following liver transplantation: incidence and predisposing factors. Clin Transpl. 2007;21(3):423-6.

5. Kurmann A, Beldi G, Vorburger SA, Seiler CA, Candinas D. Laparoscopic incisional hernia repair is feasible and safe after liver transplantation. Surg Endosc. 2010;24(6):1451-5.

6. Mekeel K, Mulligan D, Reddy KS, Moss A, Harold K. Laparoscopic incisional hernia repair after liver transplantation. Liver Transpl. 2007;13(11):1576-81.

7. Muller V, Lehner M, Klein P, Hohenberger W, Ott R. Incisional hernia repair after orthotopic liver transplantation: a technique employing an inlay/onlay polypropylene mesh. Langenbeck's Arch Surg. 2003;388(3):167-73.

8. Piardi T, Audet M, Panaro F, Gheza F, Cag M, Portolani N, et al. Incisional hernia repair after liver transplantation: role of the mesh. Transplant Proc. 2010;42(4):1244-7.

9. Piazzese E, Montalti $R$, Beltempo P, Bertelli $R$, Puviani $L$, Pacile $V$, et al. Incidence, predisposing factors, and results of surgical treatment of incisional hernia after orthotopic liver transplantation. Transplant Proc. 2004; 36(10):3097-8

10. Scheuerlein H, Rauchfuss F, Gharbi A, Heise M, Settmacher U. Laparoscopic incisional hernia repair after solid-organ transplantation. Transplant Proc. 2011:43(5):1783-9.

11. Shi LW, Verran D, Rao AR, Stewart GJ, McCaughan GW. Incisional hernia following orthotopic liver transplantation. Transplant Proc. 2003;35(1):425-6.

12. Vardanian AJ, Farmer DG, Ghobrial RM, Busuttil RW, Hiatt JR. Incisional hernia after liver transplantation. J Am Coll Surg. 2006;203(4):421-5.

13. Janssen H, Lange R, Erhard J, Malago M, Eigler FW, Broelsch CE. Causative factors, surgical treatment and outcome of incisional hernia after liver transplantation. Br J Surg. 2002;89(8):1049-54.

14. Borab ZM, Shakir S, Lanni MA, Tecce MG, MacDonald J, Hope WW, et al. Does prophylactic mesh placement in elective, midline laparotomy reduce the incidence of incisional hernia? A systematic review and meta-analysis. Surgery. 2017;161(4):1149-63.

15. Nieuwenhuizen J, Eker HH, Timmermans L, Hop WC, Kleinrensink GJ, Jeekel $J$, et al. A double blind randomized controlled trial comparing primary suture closure with mesh augmented closure to reduce incisional hernia incidence. BMC Surg. 2013;13:48 2482-13-48

16. Deeken CR, Abdo MS, Frisella MM, Matthews BD. Physicomechanical evaluation of absorbable and nonabsorbable barrier composite meshes for laparoscopic ventral hernia repair. Surg Endosc. 2011;25(5):1541-52.

17. Deeken CR, Abdo MS, Frisella MM, Matthews BD. Physicomechanical evaluation of polypropylene, polyester, and polytetrafluoroethylene meshes for inguinal hernia repair. J Am Coll Surg. 2011;212(1):68-79.

18. Martin DP, Williams SF. Medical applications of poly-4-hydroxybutyrate: a strong flexible absorbable biomaterial. Biochem Eng J. 2003;16(2):97-105.

19. Odermatt EK, Funk L, Bargon R, Martin DP, Rizk S, Williams SF. MonoMax suture: a new long-term absorbable monofilament suture made from poly4-hydroxybutyrate. Int J Polymer Sci. 2012;2012(Article ID 216137):1-12.

20. Albertsmeier $M$, Seiler CM, Fischer L, Baumann P, Husing J, Seidlmayer $C_{\text {, et }}$ al. Evaluation of the safety and efficacy of MonoMax(R) suture material for 
abdominal wall closure after primary midline laparotomy-a controlled prospective multicentre trial: ISSAAC [NCT005725079]. Langenbeck's Arch Surg. 2012;397(3):363-71.

21. Stoikes NFN, Scott JR, Badhwar A, Deeken CR, Voeller GR. Characterization of host response, resorption, and strength properties, and performance in the presence of bacteria for fully absorbable biomaterials for soft tissue repair. Hernia. 2017;21(5):771-82.

22. Deerenberg EB, Harlaar JJ, Steyerberg EW, Lont HE, van Doorn HC, Heisterkamp J, et al. Small bites versus large bites for closure of abdominal midline incisions (STITCH): a double-blind, multicentre, randomised controlled trial. Lancet. 2015;386(10000):1254-60.

23. Todd B. New CDC guideline for the prevention of surgical site infection. Am J Nurs. 2017;117(8):17.

24. General Assembly of the World Medical Association. World Medical Association Declaration of Helsinki: ethical principles for medical research involving human subjects. J Am Coll Dent. 2014;81(3):14-8.

\section{Publisher's Note}

Springer Nature remains neutral with regard to jurisdictional claims in published maps and institutional affiliations.

Ready to submit your research? Choose BMC and benefit from:

- fast, convenient online submission

- thorough peer review by experienced researchers in your field

- rapid publication on acceptance

- support for research data, including large and complex data types

- gold Open Access which fosters wider collaboration and increased citations

- maximum visibility for your research: over $100 \mathrm{M}$ website views per year

At BMC, research is always in progress.

Learn more biomedcentral.com/submissions 